\title{
LETTERS
}

\section{Higher incidence of stroke in patients with dengue fever: Spurious association or causal link?}

A recently published population-based cohort study by Li and colleagues ${ }^{1}$ suggests that patients in Taiwan with stroke were 2.49 times more likely to have received a diagnosis of dengue infection in the preceding 2 months relative to paired controls. Although uncommon, stroke associated with dengue does meet the criteria for diagnosis of severe dengue based on a guideline from the World Health Organization. ${ }^{2}$

$\mathrm{Li}$ and colleagues report an overall increase in the incidence of stroke attributable to dengue by 1.61 per 1000 personyears during 2015. If these findings are extended to all 43419 cases of dengue across Taiwan in 2015, ${ }^{3}$ then 70 cases of stroke would have occurred as a result of dengue infection. This is alarming, particularly considering that there are 96 million cases of symptomatic dengue each year worldwide. ${ }^{4}$

Have the authors identified a spurious association, or is there a genuine causal link between dengue and stroke? Comor- bidity of dengue and hemorrhagic stroke has been reported in $0.26 \%$ of cases ( 3 of 1148 ) in India, ${ }^{5}$ and $0.06 \%$ of cases (1 of 1585) in Brazil. ${ }^{6}$

Rigorous research is urgently needed to characterize the sequelae of conditions that may arise from dengue infection, including the severity and duration of underlying disease such as diabetes mellitus and hypertension, conditions that may modify the effect of dengue on the risk of stroke. If the effect of dengue on stroke is acute rather than chronic, then there must be a pathophysiologic mechanism involved. A diagnosis of severe dengue under the new classification system presents different and potentially unrelated mechanisms of action. ${ }^{7}$ In short, more clinical data are needed to support the results of Li and colleagues.

\section{Nguyen Tien Huy MD PhD}

Epidemiologist and associate professor, Department of Clinical Product

Development, Institute of Tropical

Medicine, Nagasaki, Japan

\section{Sedighe Karimzadeh}

Medical student, School of Medicine, Sabzevar University of Medical Sciences, Sabzevar, Iran

\section{R. Matthew Chico MPH PhD}

Assistant professor of public health, Department of Disease Control, London School of Hygiene \& Tropical Medicine, London, UK

Cite as: CMAJ 2019 June 17;191:E670. doi: $10.1503 / \mathrm{cmaj} .72069$

\section{References}

1. Li H-M, Huang Y-K, Su Y-C, et al. Risk of stroke in patients with dengue fever: a population-based cohort study. CMAJ 2018;190:E285-90.

2. Dengue guidelines for diagnosis, treatment, prevention and control: new edition. Geneva: World Health Organization; 2009

3. Wang S-F, Chang K, Loh E-W, et al. Consecutive large dengue outbreaks in Taiwan in 2014-2015. Emerg Microbes Infect 2016;5:e123.

4. Bhatt S, Gething PW, Brady OJ, et al. The global distribution and burden of dengue. Nature 2013;496:504-7.

5. Mathew S, Pandian JD. Stroke in patients with dengue. J Stroke Cerebrovasc Dis 2010;19:253-6.

6. de Souza LJ, Martins AL, Paravidini PC, et al. Hemorrhagic encephalopathy in dengue shock syndrome: a case report. Braz J Infect Dis 2005;9:257-61.

7. Srikiatkhachorn A, Rothman AL, Gibbons RV, et al. Dengue - how best to classify it. Clin Infect Dis 2011;53:563-7.

Competing interests: None declared. 